\title{
A GRAPHICAL INTERFACE BASED ON GRAFCET FOR PROGRAMMING INDUSTRIAL ROBOTS OFF-LINE
}

\author{
Gustavo V. Arnold*, Pedro R. Henriques \\ Department of Informatics, University of Minho \\ Braga, Portugal \\ Email: gva,prh@di.uminho.pt \\ Jaime C. Fonseca \\ Department of Industrial Electronic, University of Minho \\ Braga, Portugal \\ Email: Jaime.Fonseca@dei.uminho.pt
}

\begin{abstract}
Keywords: Compilers, Graphical Interface, Industrial Robots Programming Language, Code Generator.
Abstract: This paper presents the current development stage of our approach to industrial robot programming, that is the graphical interface for our environment, that is based on the well-known Grafcet. Our approach focus on the modelling of the system, rather than on the robot. So, it will improve the programming and maintenance tasks, allowing the reuse of source code.
\end{abstract}

\section{INTRODUCTION}

Today, there are basically two ways for programming industrial robots: (1) by the use of the industrial robot programming language (IRPL); (2) or by the use of off-line programming environments $(O L P E)$. However, both of them have their own drawbacks:

1. although the forerunner languages, such as AML (Taylor et al., 1982) or AL (Mujtaba et al., 1982), have now been superseded by elaborated robot languages, like ABB Rapid (Automation, 1994), they require detailed description of the motion of every joint in the mechanism in order to execute a desired movement; they require specialized knowledge of the language; the robot programs have limited portability, and significant investment must be made when changing or acquiring a new robot type or simply when upgrading a new controller from the same vendor.

2. the most recent off-line programming environments (for instance, Workspace) do not address the issue of sensor-guided robot actions; and they are limited to a robot motion simulator, which provides no advanced reasoning functionality, nor flexibility in the tasks.

According to some principles of software engineering and programming languages evaluating criteria

\footnotetext{
${ }^{*}$ Gustavo Arnold is a professor at Universidade Católica do Salvador - Brasil, and is temporarily at Universidade do Minho to get his PhD degree, supported by a FCT scholarship.
}

(Sebesta, 1999), it is possible to see that these development environments do not attend the user needs, as can be seen on figure 1 .

\begin{tabular}{||l||c|c||}
\hline Principles & OLPE & IRPL \\
\hline User-friendly & YES & NO \\
\hline $\begin{array}{l}\text { Source Code } \\
\text { Portability }\end{array}$ & YES & NO \\
\hline Expressiveness & NO & YES \\
\hline $\begin{array}{l}\text { Environment } \\
\text { interaction }\end{array}$ & NO & YES \\
\hline $\begin{array}{l}\text { Specification } \\
\text { close to the } \\
\text { problem }\end{array}$ & NO & NO \\
\hline Reusability & NO & NO \\
\hline
\end{tabular}

Figure 1: Principles of software engineering and programming languages that must be supported by the programming environments

So, we proposed an integrated, formal and highlevel approach to industrial robot programming (Arnold et al., 2003), that will attend these users desires. One component of this approach is a friendly graphical interface, based on the Grafcet specification diagram. This interface is responsible not only for facilitating the programming task, but also to translate this diagrammatic specification into languages that belong to different levels of our approach. This interface is user-friendly, the program specification is close to the problem (instead of close to the robot), and allows environment interaction (which means that 
our implementation will allows the development of closed-loop systems). It is also expressive, but it still can be more, depending on the other components that are under construction. The source code portability will also be achieved at the end of this implementation. And the reusability will also be achieved when extend the graphical interface.

The aim of this paper is to discuss that graphical interface based on the Grafcet, and the associated translator to RS language (Toscani, 1993) (a reactive systems programming language).

In the following sections, the most relevant theoretical aspects (like our approach to industrial robot programming, in section 2, and the Grafcet in section 3), and the components already implemented (the graphical interface in section 4, and the translator in section 5) are presented. At the end (section 6), appear the conclusions and future works.

\section{OUR APPROACH TO INDUSTRIAL ROBOT PROGRAMMING}

Our approach is described diagrammatically in figure 2. It should be supported by the following languages and tools that compose the architecture we want to defend:

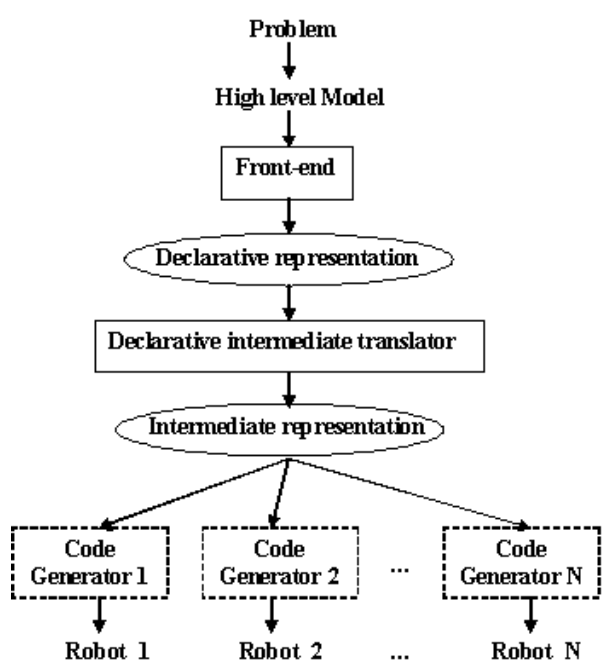

Figure 2: Proposed approach to industrial robots programming.

- a truly high level and declarative language (Grafcet, RS)

- an easy-to-use front-end

- an intermediate representation (DIR)
- the translators to RS and to DIR

- the code generator

- an automatic generator of the robot code generators

- a robot specification language

At the top, there is the problem to be solved. It will be used an adequate modelling technique, responsible for decompose the problem into simple problems, that would be easily programmed; formal models are employed to describe data structures and operations necessary to solve the subproblems. To describe formally the overall problem and the subproblems, a truly high level language, close to the specification instead of the robot, should be used. Then we advocate the use of an easy-to-use compiler front-end, like Grafcet, that can interpret the specification language and generate a declarative description, that can be translated into an intermediate description for the program specified. An intermediate representation is used because the front-end must be focused on the specification of the problem, and not on the robot. So, there will be another component responsible for translating this intermediate code into the code of a robot. Because this compiler back-end is specific for a single robot, it is necessary to have a lot of back-ends, each of them adapted to a specific target (robot's architecture and machine code). To create these code generators, an automatic generator will be used, as can be seen in figure 3. This tool, based on the known intermediate representation, and on the robot specification, that must be included someway, will produce an optimal code generator for the specified robot.

The main objective of this approach is to make the programming task easy, with the possibility of reusing the source code to program different industrial robots, allowing to explore their potentialities.

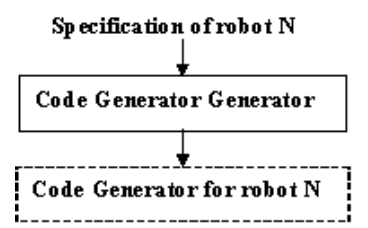

Figure 3: Automatic generator of code generators.

\section{GRAFCET}

Because Grafcet (Telemec, 1982) is a well known specification diagram for industrial automation applications, it was decided to use a graphical interface that should looks like the Grafcet, as told above.

So before describing the FE interface built, we discuss the basic concepts that Grafcet uses to represent automatisms, that are (Grafcet, 2000): 
- Step, represents a partial state of system, in which an action was performed. The step can be active or idle. The associated action is performed when the step is active, and remains asleep when the step is idle;

- Transition, links a precedent step (one or several) to a consequence step (one or several), and represents the actions flow. It describes a state change. Changing is under the control of two conditions:

1. every step previous to the transition must be active (and the actions executed),

2. a boolean condition associated with the transition, must be true.

\section{GRAPHICAL INTERFACE}

The interface that was implemented represents all the concepts and features present on a Grafcet diagram, and have a similar appearance. An example of this interface can be seen on figure 4

On this example, a robot can move throw three points, P1, P2 and P3 (P2 at the center, P1 to the left, and $\mathrm{P} 3$ to the right), and at each moment can only be in one of these positions. There are three buttons, responsible for indicating the point to where the robot must make a transfer. When one button is pressed, the robot must do the following steps:

- Close the gripper;

- Make a transfer to the a left or to the right, depending on the pressed button and the current position;

- Open the gripper when arriving at the desired position.

Based on Grafcet, this example is implemented in the following way: The step E1, that is the initial one, represents the rest state for the robot. At this moment, the robot can be in any of the $\mathrm{P} 1, \mathrm{P} 2$ or $\mathrm{P} 3$ positions. When a button (D1, D2 or D3) is pressed, a position is selected; then the robot must make a transfer from its current position to the desired one. The three possibilities (D1 pressed when the robot is on $\mathrm{P} 2$ or P3, etc) are described by the three transitions below E1. The occurrence of one of these possibilities implies a step changing to E2, E3 or E4. At any of these steps, the robot must close the gripper (Close G). After receiving the information that the gripper is closed (GC), there is another step changing, to E5, E6, E7, or E8. At any of these steps, the transfer is done and the robot moves to the left (TL) or to the right (TR). Steps E6 and E7 will be chosen depending also on the initial position of the robot (P3 or P1), because $\mathrm{P} 2$ is the central position. So if the robot is at $\mathrm{P} 1$, it must move to the right, and if it is at P3 it must move to the left. When the robot arrives at the desired position (AP1,
AP2 or AP3), another step changing is done, and the robot must open de gripper (Open $\mathrm{G}$ ). When the gripper is opened (GO), the robot returns to its rest state, the step E1 (although, the robot stays at this current position).

In the rest of this section, we explain how the Grafcet constructors were implemented in our editor.

\subsection{Steps}

The steps are represented as squares. Inside there are two fields: the number of this step, that is a mandatory field (a step number is generated automatically, but the user can modify it); and the name of the step, that is an optional field (the user can name the step to make it easy to understand its main goal). There is a checkbox, called initial, that indicates if this is an initial step. If it is, the square border becomes thicker. After inserting a new step (square) into the diagram, the editor offers two more options, that are the two buttons inside the square: the first one is the link button, responsible for linking this step to the next transition(s); the last one is the action button, responsible for associating actions to this step (figure 5). When this action button is pressed, the square is augmented, at the right side, and a text box appears, where the user can enter the actions code. The associate button inside this new right box allows to store these actions (when they are written) associated to the current step (figure 6).

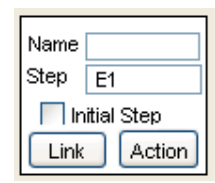

Figure 5: Step graphic representation.

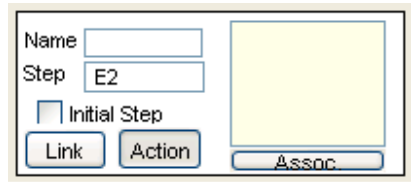

Figure 6: Step and actions graphic representation.

Each action should be written considering the following grammar, which makes possible to represent all the conditions and actions proposed by Grafcet:

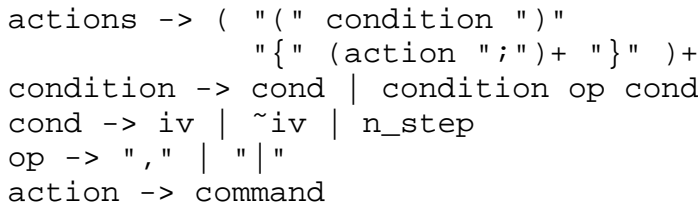




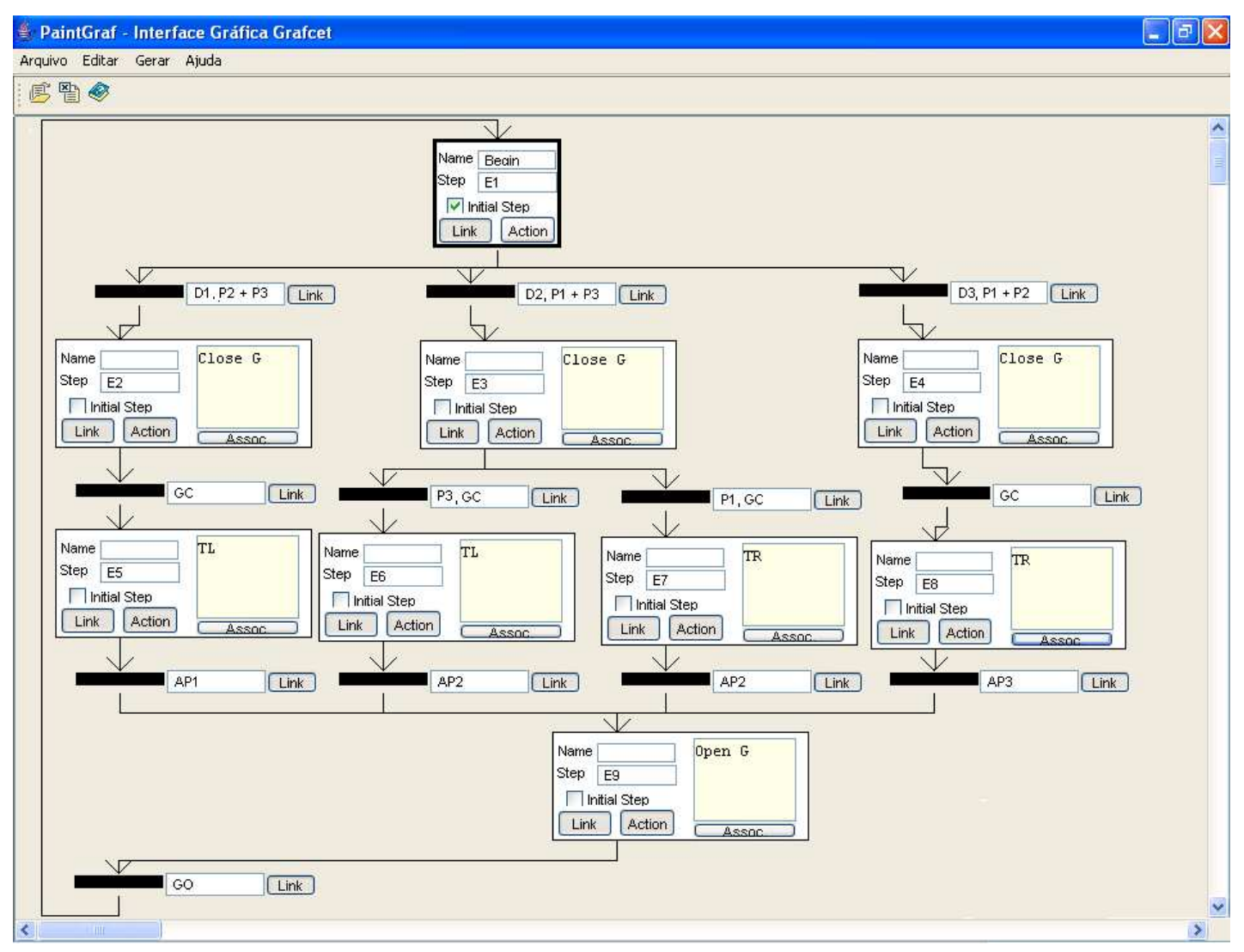

Figure 4: Graphical interface based on Grafcet.

Conditions are written inside" () " and they are optional; iv corresponds to an input variable that must be on, while the $\sim$ iv corresponds to an input variable that must be off, and n_step corresponds to steps that must be active. If a condition is satisfied, the action(s) can be executed. If there are more then one action to be executed for one condition, these actions must be written inside " \{\}$"$. It is necessary to use a ";" at the end of each action.

\subsection{Transitions}

Each transition is represented by a thick horizontal line, followed by the condition field, that must be written by the user, and by a link button, responsible for linking this transition to the next step(s) (figure 7).

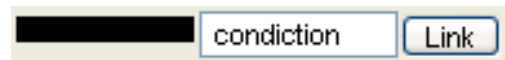

Figure 7: Transition graphic representation.

Each condition should be written considering the following grammar, which makes possible to represent all the functions proposed by Grafcet:

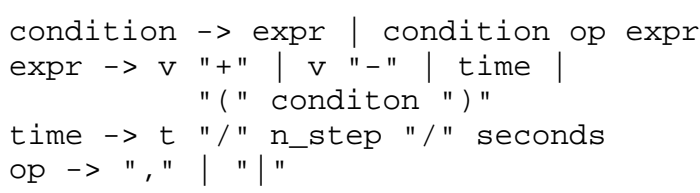

where $\mathrm{v}+$ corresponds to set the variable $\mathrm{v}$ to 1 , while the $\mathrm{v}-$ corresponds to reset the variable $\mathrm{v}$ to 0 .

\subsection{Alternatives and Simultaneous Sequences}

The sequences are generated automatically by the system. The user must only link the steps to the transitions and vice-versa. If the user wants to link one transition to more than one step, a double line is presented, to show that this is the beginning of a simultaneous sequence. Also, if the user links more than one step to only one transition, a double line is also presented, but to indicate that this is the end of a simultaneous sequence. The other links are treated as alternative sequences. Each object can be moved to any position in the window, without loosing the links. 


\section{TRANSLATOR}

At this moment, there is only one translator ready, that is the one that translates the Grafcet graphical representation into an RS program. The Grafcet elements implemented on the graphical interface were discussed on previous sections, while the RS language and automaton can be seen on (Toscani, 1993)

The variables are defined previously by the user, but the input, output and internal signals are detected automatically. The RS internal signals correspond to the steps, the RS input signals are detected during the parsing of the conditions (from actions and from transitions), and the RS output signals are detected during the parsing of the actions that must be executed.

Some auxiliary RS internal signals are also generated, if there are more then one action associated to one condition or step. They are generate to grant the sequential execution of each of these actions.

All the conditions are written using the infix notation. So, a boolean expressions analyzer was created.

It is also possible to call the RS compiler, to translate this RS program into an automaton.

To illustrate the idea, figure 8 shows the generated RS program for the sample Grafcet specification presented in figure 4. The up(signal) is responsible for activating an internal signal, and the emit(command) is responsible for sending an specific command to the environment.

\subsection{Steps}

Each step of Grafcet is treated as an internal signal of the RS language. So, for each step an internal signal is created, if it still does not exist, and included at the module header of the RS program. If the step is a initial one, it is added a command up (this step) at the initially sentence of the module header. This means that the respective internal signal will be activated at the beginning, starting the automaton execution at this step. The rules are executed if the signals (internal or input) on the left side of the rule are on. The internal signals can be signalized internally, while the input ones are set by the external environment.

If the step contains some action, it will be parsed to detect the actions and their respective conditions, if they exist. For each action, an output signal is created and inserted at the module and program headers, and an emit (this action) command is added at the point where this action should be executed. The output signals are responsible for sending commands to the external environment (as a consequence of the emit command).

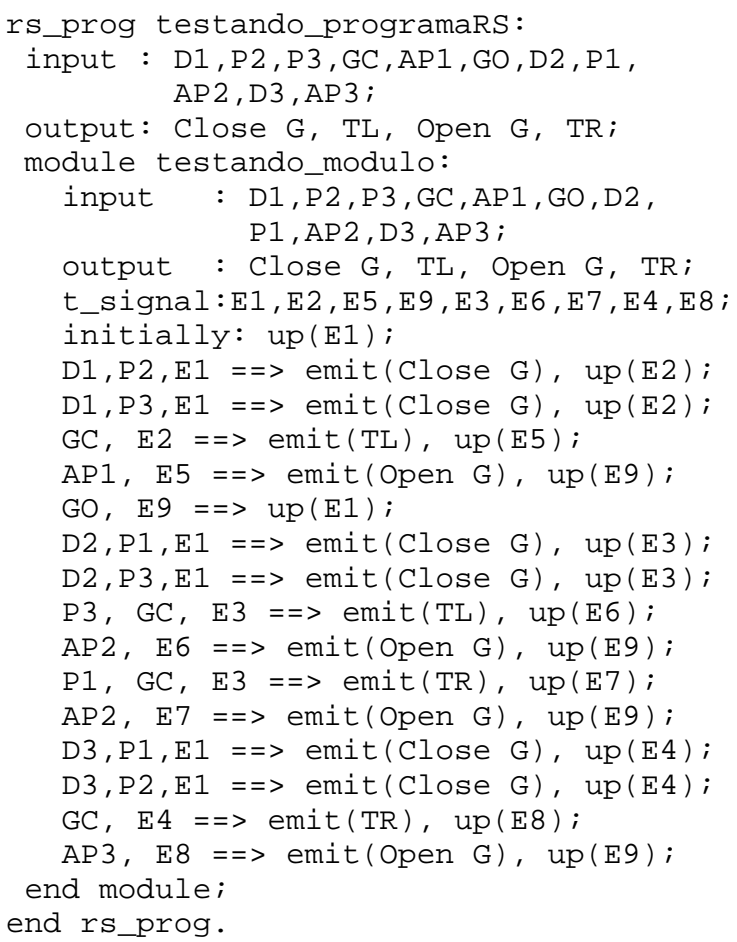

Figure 8: Example of a generated RS program.

\subsection{Transitions}

Each Grafcet transition is parsed to detect the conditions, the kind of each element (input variables, auxiliary variables, steps), and to analyze the boolean conditions that may exist. Each input variable is added as an input signal (if it does not exist) at module and program headers; each step is added as an internal signal at module heading (if it does not exist). The auxiliary variables were added directly by the user, when using the graphical interface, but they are included on the respective rule, to perform the correct behavior. All of these elements will be included on the left side of the respective rule, with the internal signals corresponding to the steps that precede this transition.

\subsection{Alternatives and Simultaneous Sequences}

The alternative sequences are detected and included at the end of each rule, by the use of the up (next step) command. This command is responsible for activating the internal signal associated to the next step that must be evaluated. There is no problem in closing this kind of sequence, because there is no synchronization between the steps of this sequence.

The simultaneous sequences are treated at the same way, but because one transition will trigger more than 
one step, it will be included more than one up (next step), to activate all the internal signals that are associated to the next steps. Because it is necessary to synchronize the last steps at the end of this sequence, each of these last steps will create an internal signal and activate it. The following step will only be executed if all the internal signals from the precedent steps are active.

\subsection{Basic Algorithm}

This section presents the basic algorithm of the translator described. We think that the algorithm is selfexplanatory and do not deserve more comments.

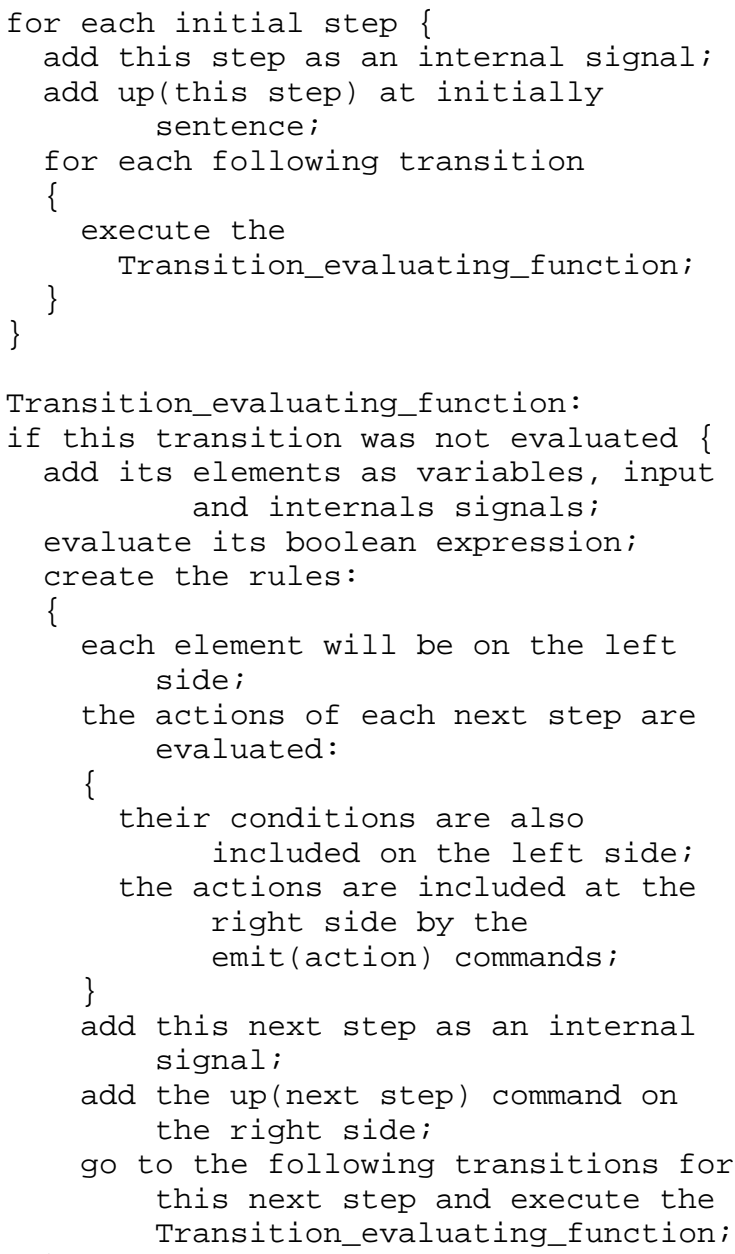

\section{CONCLUSIONS}

This paper presented a graphical interface for highlevel industrial robot programming, based on the
Grafcet; it was also introduced the respective translator from Grafcet to RS language. This work is part of an approach to industrial robot programming, that will cover all the stages of the programming task, from the modelling of the system until the robot code generation.

Both the translator and the Graphical interface were developed using Java language. Java was chosen because the goal of this project is to grant portability for the source code. So, it is important to use a language that is also platform independent.

As Grafcet is completely implemented, the programming task becomes easier. And as the RS translator generates an efficient and simple automaton, the generated code must also be efficient and simple, like the one presented at (Piola, 1998).

The next step is the development of a translator from the automaton RS to DIR, or from the automaton RS to an industrial robot language. Then we will address the development of the code generator generator.

By now, our approach satisfies the following criteria of figure 1: user-friendly, expressiveness, environment interaction, and specification close to the problem. When the implementation finishes, all of the principles in figure 1 will be granted.

\section{REFERENCES}

Arnold, G. V., Henriques, P. R., and Fonseca, J. C. (2003) A development approach to industrial robots programming. In Proceedings of the 11th International Conference on Advanced Robotics, volume 1, pages 167172, Coimbra, Portugal. Institute for Systems and Robotics, University of Coimbra.

Automation, A. F. (1994). Rapid Reference Manual 3.0.

Grafcet (2000). Grafcet homepage. http://www.lurpa.ens-cachan.fr/grafcet.html.

Mujtaba, M. S., Goldman, R., and Binford, T. (1982). Stanford's al robot programming language. Computers in Mechanical Engineering.

Piola, S. J. (1998). Uso da linguagem rs no controle do robô nachi sc15f. Trabalho de Conclusão de Curso de Graduação, Departamento de Informática, UCS, Caxias do Sul, Brasil.

Sebesta, R. W. (1999). Concepts of programming languages. Addison Wesley Longman, Inc., 4nd edition.

Taylor, R. H., Summers, P. D., and Meyer, J. M. (1982). Aml: a manufacturing language. The International Journal of Robotics Research, 1(3).

Telemec (1982). O Grafcet - Diagrama Funcional para Automatismos Sequenciais. Portugal.

Toscani, S. S. (1993). RS: Uma Linguagem para Programação de Núcleos Reactivos. Tese de doutoramento, Depto de Informática, UNL, Lisboa, Portugal. 